\title{
Influence of the Chemical Composition of Stainless Steels on the Electronic Structure of Passive Films Formed in Artificial Sea Water
}

\author{
M. Júlia J. Ferreira, ${ }^{*}$ M. da Cunha Belo ${ }^{\text {a,b }}$ \\ ISEL - Dep. Eng. Química, Rua Conselheiro Emídio Navarro, 1900 Lisboa, Portugal \\ ${ }^{a}$ IST - Dep. Eng. Química, Av. Rovisco Pais, 1049-001 Lisboa, Portugal \\ ${ }^{b}$ CNRS-CECM, 15 Rue Georges Urbain F94407, Vitry-sur-Seine Cedex, France
}

Received 12 December 2003; accepted 14 May 2004

\begin{abstract}
The electronic structure of the passive films formed on stainless steels of different chemical composition in artificial sea water is examined by capacitance measurements (Mott-Schottky approach) and photo-electrochemistry. Analytical characterization is carried out by Auger Electron Spectroscopy. The influence of the main alloying elements $(\mathrm{Cr}, \mathrm{Ni})$ on the development of space charge regions, which can be described as a depletion or accumulation layers are discussed.
\end{abstract}

Keywords: stainless steels, passive films, electronic structure.

\section{Introduction}

S'il est certain que l'augmentation de la concentration en chrome et en nickel a des effets bénéfiques vis-à-vis de la résistance à la corrosion des aciers inoxydables, les mécanismes d'action de ces éléments, en ce qui concerne le pouvoir protecteur du film passif, sont loin d'être bien compris. Cette situation résulte de l'extrême complexité des films passifs en termes de structure, composition et comportement électrochimique, lequel est illustré par la valeur du

\footnotetext{
* Corresponding author. E-mail address: jferreira@deq.isel.ipl.pt
} 
courant de passivation. Un des problèmes posés est celui de connaître comment la polarisation agit sur le transport ionique et le transfert électronique et par conséquent sur la formation des barrières de potentiel dues à l'excès de charges électriques.

La présente recherche concerne les films passifs formés dans l'eau de mer artificielle sur deux nuances d'acier inoxydable de structure austénitique lesquels diffèrent au niveau des concentrations en chrome, en nickel et en molybdène. C'est un développement important du travail effectué dans le cadre d'une recherche précédente où seule le comportement de l'acier inoxydable du type AISI 316 a été examiné [1]. L'étude est conduite à l'aide des mesures de capacité (méthode de Mott-Schottky) et de photoélectrochimie. Elle vise à préciser l'influence des principaux éléments d'alliage $(\mathrm{Cr}, \mathrm{Ni})$ sur la structure électronique des interfaces métal/film et film/électrolyte.

\section{Conditions expérimentales}

Le tableau 1 donne la composition de l'eau de mer artificielle normalisée de pH 7.5 à 8. La solution est agitée et saturée en oxygène par barbotage d'air (1.5 L/min). Le volume d'électrolyte contenu dans la cellule de mesure électrochimique et photoélectrochimique est de $150 \mathrm{~mL}$ pour une surface d'échantillons de $2 \mathrm{~cm}^{2}$. Les essais ont été effectués à la température de $25^{\circ} \mathrm{C}$. Après polissage mécanique (papier SiC 1200), lavage et nettoyage aux ultrasons, les échantillons sont séchés à l'air et subissent un traitement de surface dans le réactif $20 \% \mathrm{HNO}_{3}+2 \% \mathrm{HF}$ pour dissoudre les inclusions.

Tableau 1. Solution d'eau de mer artificielle (norme ASTM D1141).

\begin{tabular}{lc|ll}
\hline & & & \\
$\mathrm{NaCl}$ & $24.530 \mathrm{~g} / \mathrm{L}$ & $\mathrm{NaHCO}_{3}$ & $0.201 \mathrm{~g} / \mathrm{L}$ \\
$\mathrm{MgCl}_{2}, 6 \mathrm{H}_{2} \mathrm{O}$ & $11.103 \mathrm{~g} / \mathrm{L}$ & $\mathrm{KBr}_{2}$ & $0.101 \mathrm{~g} / \mathrm{L}$ \\
$\mathrm{Na}_{2} \mathrm{SO}_{4}, 10 \mathrm{H}_{2} \mathrm{O}$ & $9.278 \mathrm{~g} / \mathrm{L}$ & $\mathrm{H}_{3} \mathrm{BO}_{3}$ & $0.027 \mathrm{~g} / \mathrm{L}$ \\
$\mathrm{CaCl}_{2}, 2 \mathrm{H}_{2} \mathrm{O}$ & $1.539 \mathrm{~g} / \mathrm{L}$ & $\mathrm{SrCl}_{2}, 6 \mathrm{H}_{2} \mathrm{O}$ & $0.042 \mathrm{~g} / \mathrm{L}$ \\
$\mathrm{KCl}$ & $0.695 \mathrm{~g} / \mathrm{L}$ & $\mathrm{NaF}$ & $0.003 \mathrm{~g} / \mathrm{L}$ \\
\hline
\end{tabular}


L'analyse par spectroscopie des électrons Auger est effectuée avec l'analyseur à miroir cylindrique équipé d'un canon à électrons coaxial (Riber OPC 105). L'énergie du faisceau primaire, avec $30^{\circ}$ d'incidence avec le plan normal à la surface de l'échantillon, a été fixée à $2.5 \mathrm{KeV}$. Le faisceau d'ions krypton utilisé dans le décapage opère sous $3 \mathrm{KeV}$ et avec un angle d'incidence de $50^{\circ}$. Dans ces conditions, le taux de décapage est de $0.5 \mathrm{~nm} \mathrm{~min}^{-1}$.

Le tableau 2 donne la composition chimique des deux nuances d'alliages étudiés dans la présente recherche. Se sont des aciers inoxydables austénitiques caractérisés par des concentrations en chrome, en nickel et en molybdène très différentes. A noter que les deux matériaux sont utilisés dans les essais expérimentaux à l'état hypertrempé.

Tableau 2. Composition chimique des aciers inoxydables (\% en poids).

\begin{tabular}{ccccccccccccc}
\hline Alliage & $\mathrm{C}$ & $\mathrm{Mn}$ & $\mathrm{Si}$ & $\mathrm{P}$ & $\mathrm{S}$ & $\mathrm{Cr}$ & $\mathrm{Mo}$ & $\mathrm{Cu}$ & $\mathrm{Al}$ & $\mathrm{Ni}$ & $\mathrm{B}$ & $\mathrm{V}$ \\
1 & 0.023 & 1.19 & 0.45 & 0.027 & 0.017 & 16.40 & 1.98 & 0.46 & 0.01 & 11.01 & 0.002 & 0.061 \\
\hline Alliage & $\mathrm{C}$ & $\mathrm{Mn}$ & $\mathrm{Si}$ & $\mathrm{S}$ & $\mathrm{Cr}$ & $\mathrm{Mo}$ & $\mathrm{Cu}$ & $\mathrm{Ni}$ & $\mathrm{N}$ & & & \\
2 & 0.01 & 0.9 & 0.2 & 0.001 & 25.0 & 4.7 & 1.4 & 25 & 0,2 & & & \\
\hline
\end{tabular}

La mesure des capacités est effectuée au moyen d'un potentiostat (273A EGG/PAR) associé à une détection synchrone double phase (5208 Brookdeal) pour les mesures aux hautes fréquences; aux basses fréquences, est utilisée la technique de mesure de l'impédance par transformée de Fourrier. S'agissant des mesures de capacité, la variation du potentiel appliqué se fait dans le sens anodique-cathodique à partir de la valeur du potentiel de corrosion jusqu'à - 1.5 V/ecs. La modulation du signal perturbateur est de $3.1 \mathrm{KHz}$ ou de $13 \mathrm{~Hz}$ avec une amplitude de $10 \mathrm{mV}$ r.m.s.

L'installation utilisée dans l'étude photoélectrochimique comprend: une lampe à arc xénon de $150 \mathrm{~W}$, associée à un réflecteur et à un condenseur. L'ensemble est couplé par l'intermédiaire d'une lentille de focalisation à un monochromateur (H25, Jobin Yvon) équipé d'un réseau gravé à $1200 \mathrm{~T} / \mathrm{mm}$, blasé à $500 \mathrm{~nm}$. Les fentes d'entrée et sortie sont réglées à $2 \mathrm{~mm}$, ce qui donne une bande passante d'environ 13 nm. Le domaine spectral utilisé est de 330 à $750 \mathrm{~nm}$. Au dessus de 
$450 \mathrm{~nm}$ l'utilisation d'un filtre "long pass" permet d'éliminer les secondes harmoniques UV. Les photocourants sont mesurés par la technique de la détection synchrone. Le montage électrochimique est dans tous les cas un montage classique à trois électrodes avec une cellule comportant une fenêtre en silice transparente. Signalons que les potentiels électrochimiques sont exprimés par rapport au potentiel de l'électrode au calomel saturé (ecs).

\section{Résultats}

\section{Etude électrochimique}

La Fig. 1 montre l'évolution du potentiel de corrosion des aciers inoxydables concernés par l'étude au cours de leur immersion dans l'eau de mer artificielle. On observe que les valeurs de potentiels atteints après quelques centaines d'heures sont du même ordre de grandeur. Toutefois, la montée du potentiel est plus rapide dans le cas de l'alliage moins riche en chrome et en nickel (alliage 1).

\section{Etude par mesure des capacités}

Le comportement semi-conducteur des films passifs formés sur les aciers inoxydables est intimement lié à la capacité de la zone de charges d'espace. Cette dernière peut-être décrite par la relation de Mott-Schottky (M.S.), laquelle est donnée par l'équation suivante [1,2]:

$$
\frac{1}{C^{2}}=\frac{2}{\varepsilon \varepsilon_{0} q N_{q}}\left(U-U_{b p}+\frac{k T}{e}\right)
$$

où $N_{q}$ est la densité des porteurs $\left(\mathrm{N}_{\mathrm{d}}\right.$ pour les donneurs et $\mathrm{N}_{\mathrm{a}}$ pour les accepteurs), $\varepsilon$ la constante diélectrique du film , $\varepsilon_{0}$ la permittivité du vide, $k$ la constante de Boltzmann, $T$ la température, $U b p$ le potentiel de bandes plates et $q$ la charge élémentaire ( $\mathrm{e}^{-}$pour les électrons et $\mathrm{e}^{+}$pour les trous). 


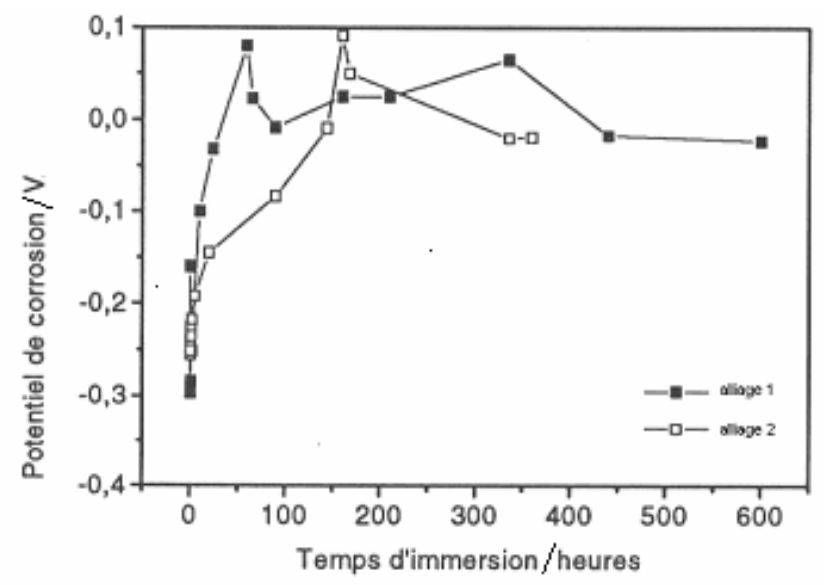

Figure 1. Influence du temps d'immersion (dans l'eau de mer artificielle) sur le potentiel électrochimique des alliages 1 et 2 .

La Fig. 2 montre l'évolution du comportement capacitif des deux aciers avec le temps d'exposition à l'eau de mer artificielle. La représentation $1 / \mathrm{C}^{2}$ en fonction du potentiel appliqué révèle l'existence de deux régions séparées par un potentiel proche de $-0.5 \mathrm{~V}$. Dans la région plus anodique $(>-0.5 \mathrm{~V})$ la capacité est représentative du comportement électrochimique d'un semi-conducteur de type $n$. En revanche le comportement capacitif observé dans le domaine des potentiels inférieurs à - $0.50 \mathrm{~V}$ est sensé décrire les propriétés d'un semi-conducteur de type p. Cette interprétation est fondée sur des recherches récentes conduites sur la passivation des aciers inoxydables (type AISI 304 et 316) et celle des éléments d'alliage ( $\mathrm{Fe}, \mathrm{Cr}, \mathrm{Ni}$ ) dans une solution tampon borate-acide borique [3]. Les films de passivation formés dans ce milieu sont alors constitués d'un duplex formé d'une zone interne d'oxyde de chrome et d'une zone externe d'oxyde de fer. Dans le cas de l'eau de mer artificielle, ce duplex, nous le verrons grâce à l'étude analytique, n'est pas bien défini. 


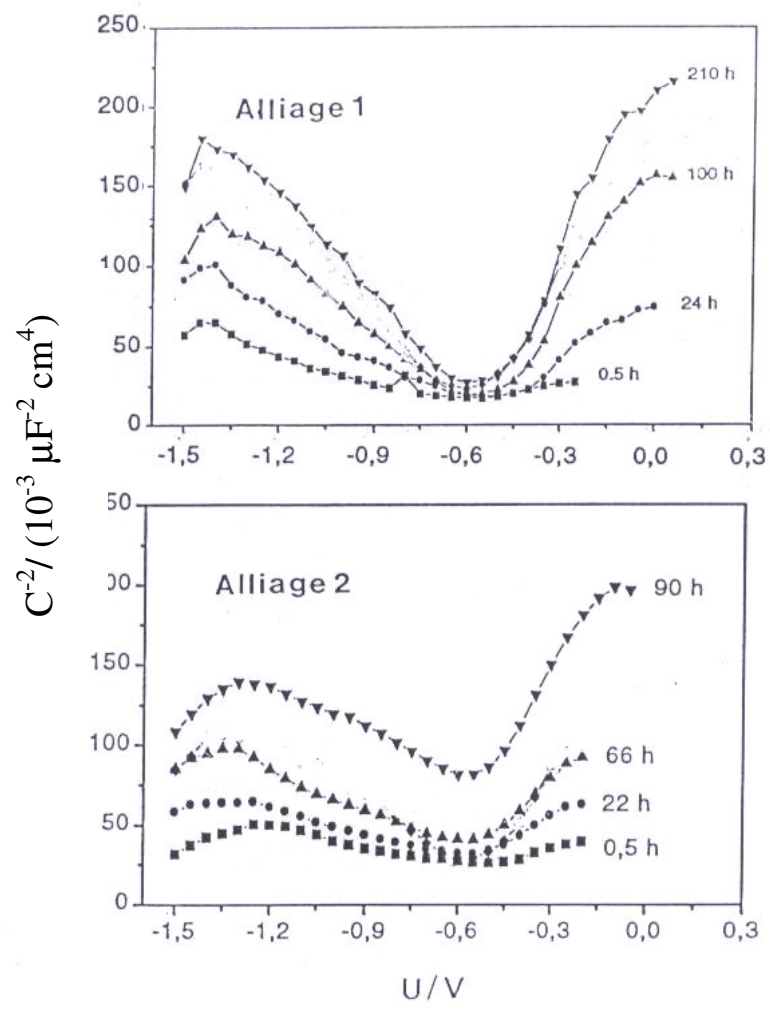

Figure 2. Représentations de $\mathrm{C}^{-2}$ en fonction du potentiel appliqué, relatives aux films passifs formés par différents temps d'immersion sur les alliages 1 et 2 .

\section{Etude photoélectrochimique}

La détermination de l'énergie de la bande interdite suppose la connaissance de la relation traduisant la variation du photocourant Iph en fonction de l'énergie des photons incidents, $h v$. Cette relation, en accord avec le modèle de Gärtner [4], est la suivante:

$$
I p h=e \Phi A W \frac{(h v-E g)^{n}}{h v}
$$

où $e$ est la charge élémentaire, $\Phi$ le flux des photons incidents, $W$ l'épaisseur de la charge d'espace, Eg l'énergie de la bande interdite et $n$ le type de transition.

On peut définir le rendement quantique $\eta$, comme étant le rapport entre le nombre de charges photo créées et le flux des photons incidents:

$$
\eta=\frac{I p h}{\Phi}
$$




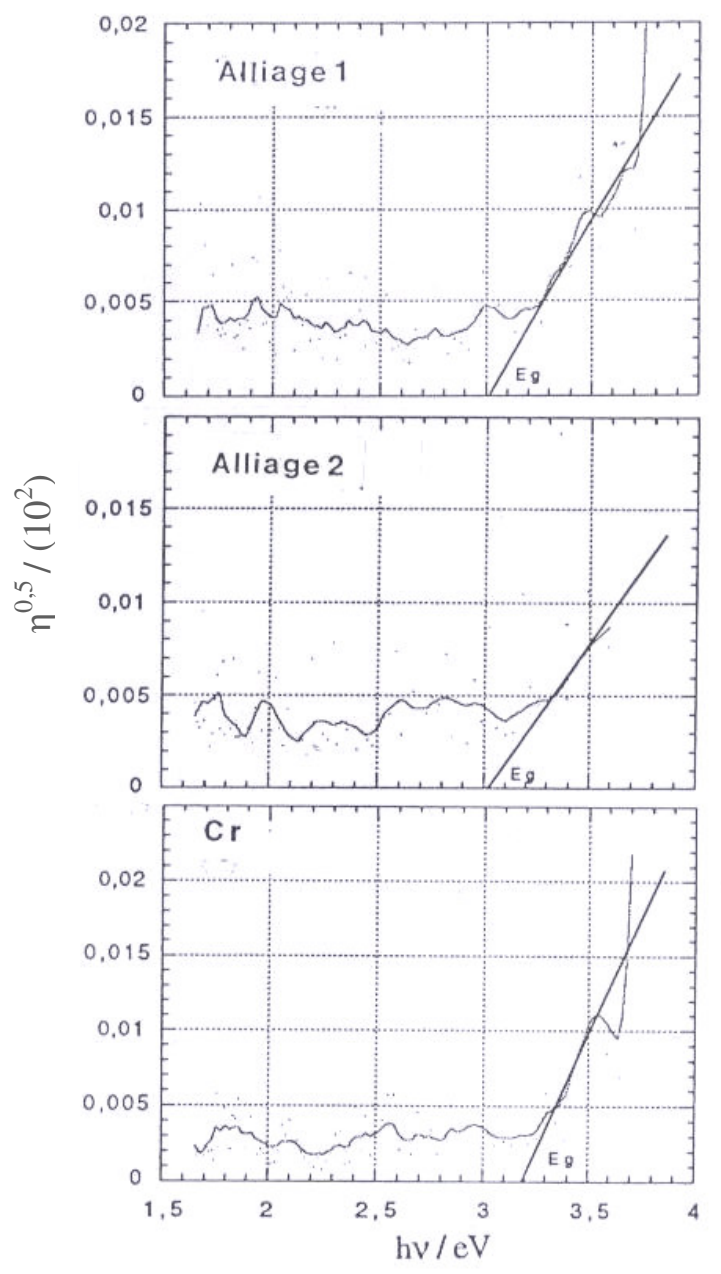

Figure 3. Rendement quantique de photo courant, obtenu avec les films passifs formés sur les alliages 1 et 2 et sur le chrome, en fonction de l'énergie des photons.

Les films de passivation formés sur les aciers inoxydables 1 et 2 sont extrêmement minces et de ce fait les photocourants créés par bombardement photonique sont très faibles. Par ailleurs le rendement quantique des oxydes de chrome est aussi très faible électrochimique.

La Fig. 3 montre que l'énergie de la bande interdite déterminée par les spectres obtenus soit avec les deux alliages, soit avec le chrome, est pratiquement la même. Elle est de l'ordre de $3.1 \mathrm{eV}$ et donc caractéristique dans les trois cas de l'oxyde de chrome.

La Fig. 4 permet de comparer la réponse capacitive à celle du photocourant. Ce dernier est anodique dans la région des potentiels plus nobles et devient 
cathodique dans la région des potentiels moins nobles. En fait, la courbe représentative du photocourant a une évolution comparable à celle de la capacité dans la représentation de M.S. Le potentiel de bandes plates déterminé par la photoélectrochimie est toutefois relativement plus anodique $(\approx 02 \mathrm{~V})$ que celui déterminé par les mesures de capacité.

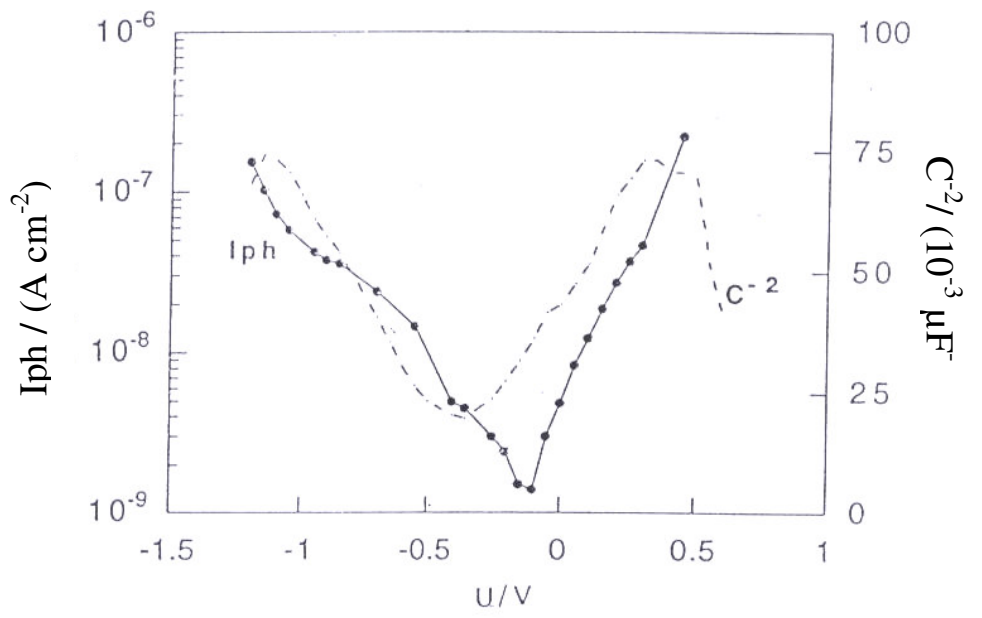

Figure 4. Influence du potentiel appliqué sur le photocourant obtenu dans le cas de l'alliage 1 préalablement passivé (16 h) dans l'eau de mer artificielle.

\section{Etude analytique par spectroscopie des électrons Auger}

Les Fig. 5 et 6 montrent les différents profils de composition chimique des films passifs formés sur les aciers inoxydables 1 et 2 .

L'approche quantitative utilisée permet l'accès aux vrais profils de composition chimique par l'utilisation du modèle de Sanz et Hoffmann (the sequential sputtering model) et d'itérations successives [5]. On suppose toutefois qu'une interface abrupte décrit l'interface vraie entre le film passif et le substrat métallique. Cette méthode analytique a été appliquée à l'étude des films de passivation formés sur différentes nuances d'aciers et alliages inoxydables [6]. L'étude effectuée dans le cadre de cette recherche montre que les films sont ultra minces (7 couches atomiques) et principalement constitués d'oxyde de chrome. Ce dernier est composé de deux couches ayant le nombre d'atomes de chrome correspondant à l'oxyde de chrome $\mathrm{Cr}_{2} \mathrm{O}_{3}$. Dans la zone externe on détecte un peu d'oxyde de fer s'il s'agit du film formé sur l'alliage 1. 


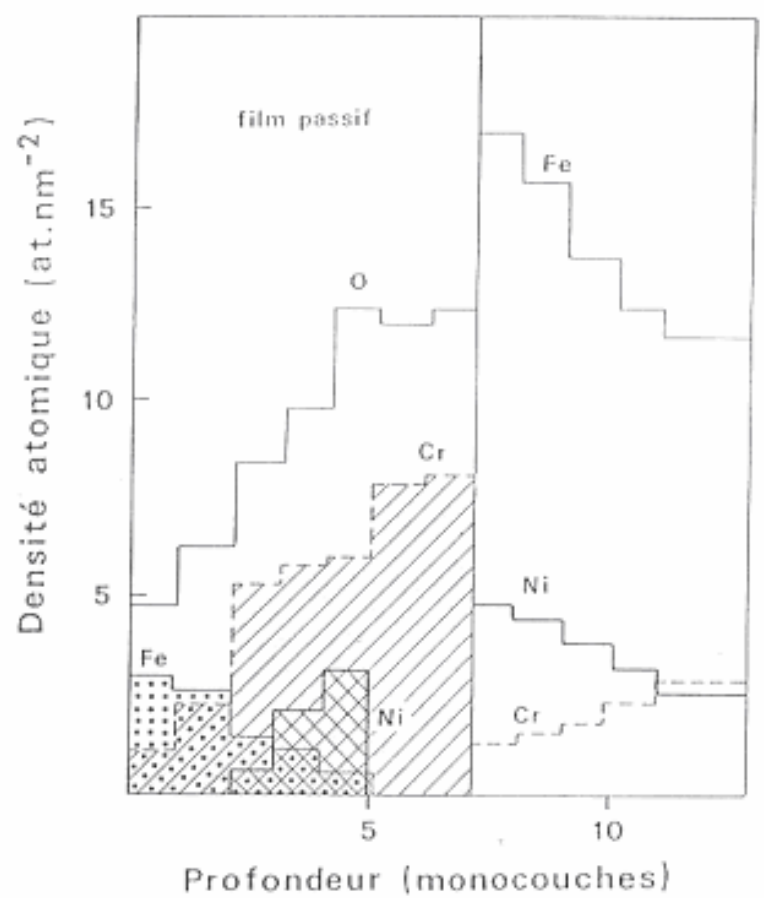

Figure 5. Profil de concentration des éléments présents dans le film formé sur l'alliage 1.

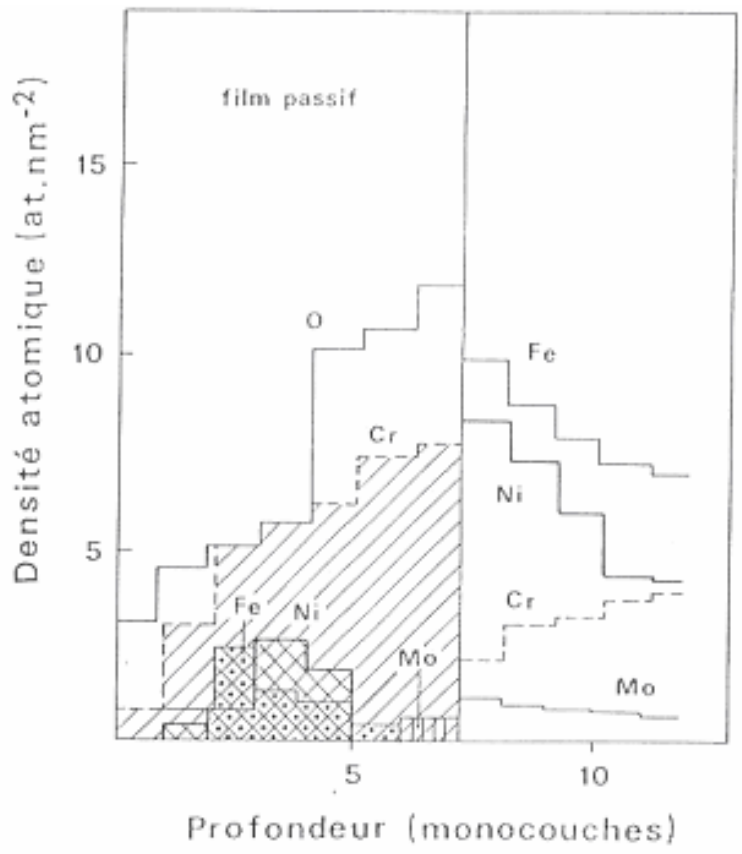

Figure 6. Profil de concentration des éléments présents dans le film formé sur l'alliage 2. 
La concentration maximale de l'oxyde de nickel apparaît située entre les oxydes de fer et de chrome. On observe par ailleurs que le substrat métallique est fortement enrichi en fer et en nickel, mais au contraire appauvri en chrome. Le film formé sur l'alliage 2 contient moins d'oxyde de fer dans la zone externe du film et inversement un peu plus d'oxyde de nickel. Le molybdène n'est pas détecté dans le film même quand sa concentration dans l'acier est élevée.

\section{Discussion}

Le comportement capacitif des films passifs formés sur les aciers inoxydables peut en général s'expliquer par le fait qu'ils sont constitués par des zones de semi-conductivités distinctes ( $n$ et $p$ ). Le changement de type de conduction s'opère dans la transition des couches internes vers les couches externes du film passif et résulte de la formation de phases également distinctes. Le film est alors formé d'un duplex ayant une couche interne d'oxyde de chrome de type $p$ et d'une couche externe d'oxyde de fer de type $n$. Un tel duplex peut se développer dans un électrolyte où l'oxyde de fer est stable, comme c'est le cas du film passif formé, sans traitement préalable, dans les solutions tampons acide borique-borate utilisées dans la présente recherche [3].

Rappelons que sur le plan cristallographique, le changement de semiconductivité correspond au passage d'un spinelle direct (chromite) à un spinelle inverse (magnétite). En fait, la grande difficulté réside dans la détermination de la semi-conductivité des oxydes mixtes.

On sait que la présence de fer dans la maille cristallographique de l'oxyde de chrome impose une conductivité de type $n$ dès que la concentration en fer devient supérieure à 5\% [7]. Signalons aussi que la formation d'oxydes où le chrome possède un état d'oxydation plus élevé peut également agir sur le mode de conduction [8]. L'existence de zones de semi-conductivité distinctes suppose aussi l'existence de jonctions, lesquelles en raison de la très faible épaisseur du film ont besoin d'être assimilées à des interfaces concernant quelques couches atomiques. Rappelons que, sans être dégénérés, les oxydes constitutifs de ces jonctions sont hautement dopés. Par ailleurs, en raison des processus de transport 
ionique, ces jonctions sont la source de défauts, c'est à dire que la distribution du potentiel électrique dans la zone de la jonction résulte des équilibres électroniques et ioniques. Notons enfin, que le changement de conductivité peut se faire tout simplement par la variation de la stoechiométrie des couches atomiques superficielles.

Dans les oxydes, le développement des charges de surface s'explique par le fait que l'enthalpie de formation des espèces cationiques est différente de l'enthalpie de formation des espèces anioniques. Si ces espèces sont les lacunes de chrome et les lacunes d'oxygène et que l'enthalpie libre, $\Delta \mathrm{G}_{\mathrm{Cr}}$, pour former une lacune de chrome est supérieure à l'enthalpie libre $\Delta \mathrm{G}_{0}$ pour former une lacune d'oxygène. Alors, on aura tendance à former un excès de lacunes cationiques en surface. Cette analyse concerne l'interface film-oxygène. S'agissant de l'interface filmélectrolyte, la concentration des espèces anioniques et cationiques est dictée par le potentiel électrochimique, $U$, lequel est en relation avec l'énergie de formation de ces espèces par la classique expression [9]

$$
U=-\frac{\Delta G_{n}}{Z F}
$$

où $\Delta G_{n}$ est l'enthalpie libre de formation de chaque espèce ionique et $F$ la quantité d'électricité appelée Faraday. $U$ est positif puisque $\Delta G_{n}$ est négatif pour toute transformation chimique spontanée.

En écrivant l'égalité du potentiel électrochimique, c'est-à-dire du potentiel de Fermi, de chaque espèce en tout point du système métal-film-électrolyte, on obtient les équations donnant les concentrations en surface, soit des lacunes anioniques $\left(V_{O}\right)$ soit des lacunes cationiques $\left(V_{M}\right),[10]$.

$$
\begin{gathered}
{\left[V_{M}\right]_{S}=\left[V_{M}\right]_{\infty} \exp \left[\frac{-2 q \varphi}{k T}\right]} \\
{\left[V_{O}\right]_{S}=\left[V_{O}\right]_{\infty} \exp \left[\frac{2 q \varphi}{k T}\right]}
\end{gathered}
$$


où $\varphi$ est le potentiel électrostatique.

Dans la partie interne du film $\left[V_{M}\right]_{\infty}=\left[V_{O}\right]_{\infty}$ du fait de l'équilibre de Schottky. En surface, leur concentration varie avec le potentiel imposé de manière symétrique par rapport au potentiel où les concentrations des lacunes cationiques et anioniques deviennent égales. La variation du potentiel électrique $\varphi$ avec la distance à la surface s'obtient en résolvant l'équation de Poisson :

$$
\Delta \varphi=\frac{[e]}{\varepsilon \varepsilon_{0}}\left(\left[V_{O}\right]-\left[V_{M}\right]\right)
$$

On trouve une variation à peu près exponentielle identique à la loi de DebyeHückel pour les électrolytes. Les mesures de capacités effectuées sur les aciers passivés dans l'eau de mer artificielle indiquent que l'égalité entre la concentration des lacunes cationiques et anioniques s'effectue aux environs du potentiel de $0 \mathrm{~V}$. Notons que ce potentiel est celui à partir duquel le courant de corrosion du chrome passivé augmente.

\section{Influence de la composition chimique de l'acier inoxydable sur la structure électronique du film passif}

Les films formés sur les alliages concernés par la présente recherche, sont essentiellement constitués d'oxyde de chrome. La plus grande différence sur le plan de l'analyse Auger réside dans la composition chimique des couches atomiques superficielles (Figs. 5-6). La présence en surface d'un oxyde mixte, avec une concentration en fer plus marquée, détermine pour le film passif formé sur l'alliage 1, l'existence d'un potentiel de bandes plates situé à environ $-0.5 \mathrm{~V}$, lequel est le potentiel d'équilibre électrochimique du fer. Le comportement de type $n$ observé entre -0.5 et $0 \mathrm{~V}$ est, en partie, provoqué par la présence en surface de ces couches d'oxyde mixte contenant du fer.

En accord avec les résultats analytiques (Fig. 6), l'influence de l'oxyde de fer apparaît nettement moins marquée dans le cas du film formé sur l'alliage 2 et de ce fait, l'existence d'un potentiel de bandes plates situé à $-0.5 \mathrm{~V}$ est plus discutable. En réalité, dans cette région des potentiels électrochimiques les 
mesures de capacité dénoncent la présence en surface des oxydes de chrome et de nickel lesquels ont un potentiel de bandes plates proche de $0.5 \mathrm{~V}$. La situation est toutefois complexe car nous avons à considérer non seulement la présence de lacunes de $\mathrm{Cr}$ et d'oxygène, mais aussi celle des interstitiels cationiques $\left(\mathrm{Fe}^{2+}\right)$. Les études concernant le structure électronique des films passifs formés dans la solution borate-acide borique de $\mathrm{pH}=9.2$, montrent que les films ont un comportement semi-conducteur, soit de type $n$ soit de type $p$ selon que le domaine de potentiels considéré est supérieur où inférieur au potentiel de bandes plates situé à environ - $0.5 \mathrm{~V}$ [3]. Ce comportement est dû aux propriétés semiconductrices des oxydes constitutifs de fer (type $n$ ) et de chrome (type $p$ ). Le modèle de structure électronique proposé signale l'existence d'une hetérojonction $p$ - $n$ située entre les couches internes d'oxyde de chrome et les couches externes d'oxyde de fer. Ce modèle s'applique aux films passifs formés sur les aciers inoxydables soit ferritiques, c'est-à-dire sur les alliages binaires Fe-Cr, soit austenitiques s'agissant des alliages Fe-Cr-Ni. Sa généralisation exige, en particulier, la connaissance du comportement capacitif des principaux éléments d'alliage.

Dans la Fig. 7, les représentations schématiques de $\mathrm{C}^{-2}=f(\mathrm{U})$ concernent les films passifs formés sur les éléments $\mathrm{Fe}, \mathrm{Cr}$ et $\mathrm{Ni}$, dans la solution de borate $(\mathrm{pH}$ 9.2) au potentiel de $0.3 \mathrm{~V}$. Pour chaque élément la réponse capacitive détermine les situations soit de déplétion soit d'accumulation. Les barrières de potentiel électrique dans la zone de la déplétion, sont formées dans le cas du fer par polarisation anodique vis-à-vis du potentiel de bandes plates $(-0.5 \mathrm{~V})$. En revanche, ces mêmes barrières sont formées dans le cas des oxydes de chrome où de nickel par polarisation dans le sens des potentiels négatifs vis-à-vis du potentiel de bandes plates lequel est situé aux environs de $0.5 \mathrm{~V}$.

S'agissant du comportement capacitif du film formé sur un acier inoxydable FeCr, dans la solution de borate, le potentiel de bandes plates apparaît situé à - $0.5 \mathrm{~V}$ [3]. Ce comportement provoqué par la formation d'un film passif constitué d'un duplex, indique que la charge d'espace développée à l'interface 
film-substrat métallique est en situation d'accumulation dans la presque totalité du domaine caractéristique de l'état passif.

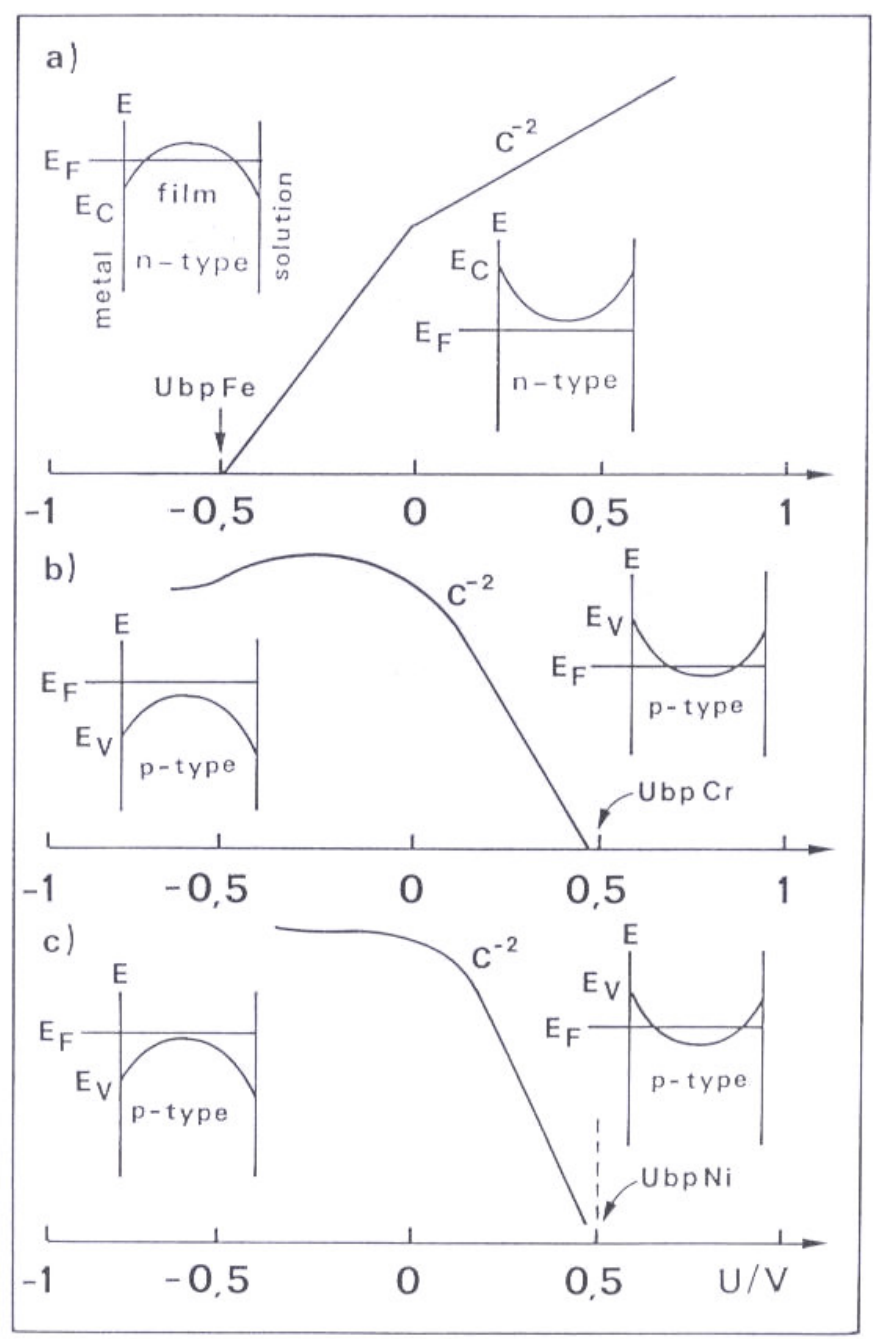

Figure 7. Représentation schématique de la capacité en fonction du potentiel appliqué (représentation de Mott-Schottky) et modèle de structure de bandes correspondant à la polarisation vis-à-vis du potentiel de bande plate, $U_{b p}$. a) Passivation du fer. b) Passivation du chrome. c) Passivation du nickel.

La réponse capacitive du film passif formé sur l'alliage 1 dans l'eau de mer artificielle est en partie comparable à celle du film passif formé sur le même alliage dans la solution de borate ( $\mathrm{pH}$ 9.2). Dans ce cas, la présence d'une faible quantité d'oxyde de fer dans les couches superficielles du film (comme le montre la Fig. 5), détermine l'existence du potentiel de bandes plates et du type de semi- 
conductivité caractéristiques de cet oxyde. En revanche, au potentiel voisin de 0 $\mathrm{V}$, la capacité est contrôlée par les processus de dissolution et formation de défauts en accord avec les équations (5) et (6).

Dans la région proche du potentiel de bande plates de l'oxyde de fer $(\sim-0.5 \mathrm{~V})$, la valeur des capacités est globalement plus faible s'agissant de l'alliage 2. Cette situation peut être attribuée au fait que cet acier contient plus de nickel. L'action de cet élément apparaît très clairement en comparant le comportement de l'acier du type AISI 304 soit à celui d'un alliage à base nickel du type Alliage 600 (Ni$16 \% \mathrm{Cr}-8 \% \mathrm{Fe})$, soit celui du nickel pur [11]. Il a été montré que la valeur minimale de $\mathrm{C}^{-2}$ relative à l'alliage coïncide avec celle de $\mathrm{C}^{-2}$ obtenue avec le nickel passivé. Ceci indique que la capacité mesurée dans la région où se situent les potentiels de bandes plates est dans ce cas équivalente à celle de l'oxyde de nickel.

Ceci montre qu'au potentiel de bandes plates, l'oxyde de nickel rend les films formés sur l'alliage 2 moins conducteurs comparativement à ceux formés sur l'acier inoxydable 1.

En revanche, pour l'alliage 2 c'est seulement dans la région de potentiels appartenant au domaine de la transpassivation du chrome, que les zones de charge d'espace développées aux interfaces métal/film et film/électrolyte, sont en situation d'accumulation.

\section{Influence de la Composition Chimique des Aciers Inoxydables sur la Structure Electronique des Films Passifs Formés dans l'Eau de Mer Artificielle}

\section{Resumé}

L'étude concerne la structure électronique des films passifs formés dans l'eau de mer artificielle, sur deux nuances d'acier inoxydable. Elle vise plus précisément à établir l'influence des principaux éléments d'alliage $(\mathrm{Cr}, \mathrm{Ni})$ sur les situations soit de déplétion soit d'accumulation dans les zones de charge d'espace formées par la polarisation aux interfaces métal/filme et film/électrolyte. Le travail expérimental repose sur les mesures de capacité (méthode de Mott-Schottky) et de photoélectrochimie. La caractérisation analytique des films passifs a été faite par spectroscopie des électrons Auger.

Mots Clés: aciers inoxydables; films passifs; structure électronique. 


\section{References}

1. $\quad$ N.F. Mott, Proc. Roy. Soc., London, Ser. A-171 (1939) 27.

2. W. Schottky, Z. Phys. Chem. 113 (1939) 376; 118 (1942) 539.

3. N.E. Hakiki, S. Boudin, B. Rondot, M. da Cunha Belo, Corros. Sci. 37 (1995) 1809-1822.

4. W.W. Gartner, Phys. Rev. 116 (1959) 84-87.

5. G. Lorang, M. da Cunha Belo, A.M. Simões, M.G.S. Ferreira, J. Electrochem. Soc. 141 (1994) 3347-3356.

6. J.M. Sanz, S. Hoffmann, Surf. Interface Anal. 8 (1986) 147.

7. W.B.A. Sharp, Corros. Sci. 10 (1970) 283.

8. M.M. Hukovic, M.C. Ceric, J. Electrochem. Soc. 134 (1987) 2193-2197.

9. K.J. Vetter, J. Electrochem. Soc. 110 (1963) 597-605.

10. F.A. Kröger, The Chemistry of Imperfections, Amsterdam North-Holland Publ. Co., 1964.

11. M. da Cunha Belo, N.E. Hakiki, M.G.S. Ferreira, Electrochimica Acta, 44 (1999) 2473. 